TITLE:

\title{
A note on submodular function minimization by Chubanov's LP algorithm
}

\author{
$\operatorname{AUTHOR}(\mathrm{S}):$ \\ Fujishige, Satoru
}

CITATION:

Fujishige, Satoru. A note on submodular function minimization by Chubanov's LP algorithm. Discrete Optimization 2019, 33: 140-145

ISSUE DATE:

2019-8

URL:

http://hdl.handle.net/2433/243228

\section{RIGHT:}

(c) 2019. This manuscript version is made available under the CC-BY-NC-ND 4.0 license

http://creativecommons.org/licenses/by-nc-nd/4.0/; The full-text file will be made open to the public on 1 August 2021, in accordance with publisher's 'Terms and Conditions for Self-Archiving'.; This is not the published version. Please cite only the published version.; この論文は出版社版でありません。引用の際には出版社版をご確認ご利用ください。 


\title{
A Note on Submodular Function Minimization by Chubanov's LP Algorithm
}

\author{
Satoru Fujishige ${ }^{1}$ \\ Research Institute for Mathematical Sciences, Kyoto University, Kyoto 606-8502, Japan
}

\begin{abstract}
Recently Dadush, Végh, and Zambelli (2017) has devised a polynomial submodular function minimization (SFM) algorithm based on their LP algorithm. In the present note we also show a weakly polynomial algorithm for SFM based on the recently developed linear programming feasibility algorithm of Chubanov (2017) to stimulate further research on SFM.
\end{abstract}

Keywords: Submodular function minimization, base polyhedra, Chubanov's algorithm 2000 MSC: 90C27, 52B40, 52A41, 90C05

\section{Introduction}

Since the advent of polynomial combinatorial algorithms for submodular function minimization (SFM) in 1999 [9, 12] substantial improvements over the algorithms have been achieved as an active area of research. From the point of view of the computational complexity the currently best SFM algorithm is obtained by Lee, Sidford, and Wong [10]. On the other hand, important developments in linear programming algorithms have recently been made by Chubanov [2, 3, 4], and Dadush, Végh, Zambelli [5] among others. Recently Dadush, Végh, and Zambelli [6] has also devised a polynomial SFM algorithm based on their LP algorithms.

The aim of this note is to give yet another approach to SFM by using Chubanov's LP algorithm [4], which should stimulate further research on SFM and hopefully lead us to some efficient algorithms both practically and theoretically.

\section{Definitions and Preliminaries}

Let $\mathbb{R}$ be the set of reals, $\mathbb{Z}$ that of integers, $\mathbb{Z}_{\geq 0}$ that of nonnegative integers, and $\mathbb{Z}_{>0}$ that of positive integers.

Let $V$ be a finite nonempty set of cardinality $|V|=n$. For any $x, y \in \mathbb{R}^{V}$ define $\langle x, y\rangle=\sum_{v \in V} x(v) y(v)$. Consider an arbitrary bounded polyhedron $Q \subset \mathbb{R}^{V}$. Denote by $\operatorname{Ext}(Q)$ the set of all extreme points of $Q$ and define

$$
\mathrm{C}^{*}(Q)=\left\{x \in \mathbb{R}^{V} \mid\langle z, x\rangle \geq 0 \text { for all } z \in \operatorname{Ext}(Q)\right\} .
$$

Then we have the following.

\footnotetext{
Email address: fujishig@kurims.kyoto-u.ac.jp (Satoru Fujishige)

${ }^{1}$ The author is very grateful to Tomonari Kitahara of Kyushu University for pointing out an error in an earlier version of this note and to the reviewers for useful comments; especially to one of the reviewers for pointing out a critical error in the use of Chubanov's algorithm. The present research is supported by JSPS Grant-in-Aid for Scientific Research (B) 25280004.
}

Proposition 1. For any bounded polyhedron $Q \subset \mathbb{R}^{V}$, the cone $\mathrm{C}^{*}(Q)$ defined by (1) is full-dimensional, i.e., of dimension $n=$ $|V|$ if and only if $\mathbf{0} \notin Q$ or $\mathbf{0} \in \operatorname{Ext}(Q)$.

Moreover, there exists a non-zero $x \in \mathrm{C}^{*}(Q)$ if and only if $\mathbf{0}$ is not in the interior of $Q$.

It should be noted that if $Q$ is not full-dimensional, then the interior of $Q$ is empty, so that there always exists a non-zero $x \in \mathrm{C}^{*}(Q)$.

Let $f: 2^{V} \rightarrow \mathbb{R}$ be a submodular function, i.e.,

$$
f(X)+f(Y) \geq f(X \cup Y)+f(X \cap Y) \quad(\forall X, Y \subseteq V),
$$

where we assume $f(\emptyset)=0$. (See [7] for more information about relevant definitions and basic results on submodular functions.)

The submodular polyhedron $\mathrm{P}(f) \subset \mathbb{R}^{V}$ associated with the submodular function $f: 2^{V} \rightarrow \mathbb{R}$ is given by

$$
\mathrm{P}(f)=\left\{x \in \mathbb{R}^{V} \mid \forall X \subseteq V: x(X) \leq f(X)\right\}
$$

and the base polyhedron $\mathrm{B}(f)$ by

$$
\mathrm{B}(f)=\left\{x \in \mathbb{R}^{V} \mid x \in \mathrm{P}(f), x(V)=f(V)\right\},
$$

where for any $X \subseteq V$ we define $x(X)=\sum_{v \in X} x(v)$ and $x(\emptyset)=0$.

We consider the membership problem of discerning whether $\mathbf{0} \in \mathrm{B}(f)$ or not and to find a set $X \subseteq V$ with $f(X)<0$ if $\mathbf{0} \notin \mathrm{B}(f)$, where $\mathbf{0}$ is the zero vector in $\mathbb{R}^{V}$. Since $f(V) \neq 0$ implies $\mathbf{0} \notin \mathrm{B}(f)$, we assume $f(V)=0$ when we consider the membership problem, unless otherwise stated. Moreover, we assume without loss of generality

( $\left.\mathbf{A}^{*}\right) \mathbf{B}(f)$ is full-dimensional, i.e., the dimension of $\mathbf{B}(f)$ is equal to $|V|-1(=n-1)$,

where note that $\mathrm{B}(f)$ lies within the hyperplane $x(V)=0 .^{2}$ This assumption is to guarantee that the maximum dimension of $W$

\footnotetext{
${ }^{2}$ Note that we can find connected components of the submodular system $\left(2^{V}, f\right)$ in $\mathrm{O}\left(n^{2} \mathbf{E O}\right)$ time by using the algorithm of Bixby, Cunningham, and Topkis [1] and each connected component has a full-dimensional base polyhedron. See [7, Section 3.3]. Here EO denotes the time required for the function evaluation of $f$.
} 
appearing in (13) is $n-1$. We thus consider the relative topology within the hyperplane $x(V)=0$.

For any $\alpha \geq 0$ define $f^{(\alpha)}: 2^{V} \rightarrow \mathbb{R}$ by

$$
f^{(\alpha)}(X)= \begin{cases}f(X)+\alpha & \text { if } X \in 2^{V} \backslash\{\emptyset, V\} \\ f(X)(=0) & \text { if } X \in\{\emptyset, V\} .\end{cases}
$$

We can easily see that $f^{(\alpha)}$ is a submodular function, which is called the $\alpha$-enlargement $t^{3}$ of $f$. It should be noted that $\mathrm{B}\left(f^{(\alpha)}\right)$ is full-dimensional, i.e., of dimension $n-1$, since $\mathrm{B}\left(f^{(\alpha)}\right) \supseteq \mathrm{B}(f)$ and $\mathrm{B}(f)$ is full-dimensional under Assumption $\left(\mathbf{A}^{*}\right)$. It should be noted that the operation of enlargement is also employed by Dadush, Végh, and Zambelli [6] and plays an important rôle in their algorithm as well.

Define vectors $\bar{\alpha}, \underline{\alpha} \in \mathbb{R}^{V}$ by

$$
\bar{\alpha}(v)=f(\{v\}), \quad \underline{\alpha}(v)=f(V)-f(V \backslash\{v\}) \quad(\forall v \in V) .
$$

(See [7, (3.89) and (3.95)]). Then we have

$$
\underline{\alpha}(X) \leq x(X) \leq f(X) \leq \bar{\alpha}(X) \quad(\forall x \in \mathrm{B}(f), \forall X \subseteq V) .
$$

Note that for any base $b \in \mathrm{B}(f)$ we must have $\underline{\alpha} \leq b \leq \bar{\alpha}$, i.e., $\mathrm{B}(f) \subseteq[\underline{\alpha}, \bar{\alpha}]$ (a standard box between $\underline{\alpha}$ and $\bar{\alpha})$.

Lemma 2. We have

$$
0 \geq \min \{f(X) \mid X \subseteq V\} \geq \sum_{v \in V} \min \{0, f(V)-f(V \backslash\{v\})\} .
$$

Moreover, for any base $b \in \mathrm{B}(f)$ we have

$$
|b(v)| \leq \max \{f(\{v\}),-f(V)+f(V \backslash\{v\})\} \quad(\forall v \in V) .
$$

Proof: The present lemma follows from (6) and (7).

The base polyhedron $\mathrm{B}(f)$ lies in the subspace $H_{0}=\{x \in$ $\left.\mathbb{R}^{V} \mid x(V)=0\right\}$. Hence, considering the relative topology in the subspace $H_{0}$, we define

$\mathrm{C}^{*}(\mathrm{~B}(f))=\left\{x \in \mathbb{R}^{V} \mid x(V)=0,\langle b, x\rangle \geq 0\right.$ for all $\left.b \in \operatorname{Ext}(\mathrm{B}(f))\right\}$.

We say $\mathrm{C}^{*}(\mathrm{~B}(f))$ is full-dimensional if it is of dimension $n-1$.

\section{Membership in Base Polyhedra and Chubanov's LP Al- gorithm}

In the sequel we assume that $f$ is an integer-valued submodular function. Let us consider the membership problem of $\mathbf{0} \in \mathbf{B}(f)$. Applying Chubanov's LP Algorithm [4], we have the following two:

1. When $\mathrm{C}^{*}(\mathrm{~B}(f))$ is full-dimensional, we can obtain a nonzero vector in $\mathrm{C}^{*}(\mathrm{~B}(f))$ in polynomial time.

2. If in prescribed polynomial time we cannot obtain such a non-zero vector in $\mathrm{C}^{*}(\mathrm{~B}(f))$, then we can conclude that $\mathrm{C}^{*}(\mathrm{~B}(f))$ is not full-dimensional, which under Assumption ( $\left.\mathbf{A}^{*}\right)$ implies $\mathbf{0} \in \mathrm{B}(f) \backslash \operatorname{Ext}(\mathrm{B}(f))$ (see Proposition 1).

\footnotetext{
${ }^{3}$ See $[7$, Section $3.1(d)]$
}

When we conclude $\mathbf{0} \in \mathrm{B}(f) \backslash \operatorname{Ext}(\mathrm{B}(f))$, we see that $f(X) \geq$ 0 for all $X \subseteq V$.

On the other hand, when we find a non-zero vector $w \in$ $\mathrm{C}^{*}(\mathrm{~B}(f)$, we can get a nonempty proper subset $Z \subset V$ with $f(Z) \leq 0$ as follows. This is a standard technique but we show it for completeness (cf. [6, Lemma 3.2]). Letting $b^{*}$ be the greedy solution that minimizes $\langle w, x\rangle$ over $\mathrm{B}(f)$, we have $\left\langle w, b^{*}\right\rangle \geq 0$ since $w \in \mathrm{C}^{*}\left(\mathrm{~B}(f)\right.$. Let $\left(v_{1}, \cdots, v_{n}\right)$ be a linear ordering of $V$ that gives the greedy solution $b^{*}$, so that

$$
w\left(v_{1}\right) \leq \cdots \leq w\left(v_{n}\right) .
$$

Putting $S_{i}=\left\{v_{1}, \cdots, v_{i}\right\}$ for $i=1, \cdots, n$ and $S_{0}=\emptyset$, we have

$$
\begin{aligned}
\left\langle w, b^{*}\right\rangle= & w\left(v_{1}\right)\left(f\left(S_{1}\right)-f\left(S_{0}\right)\right)+\cdots \\
& +w\left(v_{n}\right)\left(f\left(S_{n}\right)-f\left(S_{n-1}\right)\right) \\
= & \left(w\left(v_{1}\right)-w\left(v_{2}\right)\right) f\left(S_{1}\right)+\cdots \\
& +\left(w\left(v_{n-1}\right)-w\left(v_{n}\right)\right) f\left(S_{n-1}\right) \\
\geq & 0 .
\end{aligned}
$$

Since at least one inequality in (11) holds with strict inequality, it follows from (11) and (12) that for some $i^{*} \in\{1, \cdots, n-1\}$ we have $f\left(S_{i^{*}}\right) \leq 0$ (with $w\left(v_{i^{*}}\right)-w\left(v_{i^{*}+1}\right)<0$ ). We thus obtain a required $Z=S_{i^{*}}$.

Summing up the above arguments, we get the following theorem.

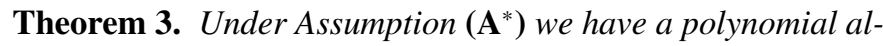
gorithm that gives us

(a) a nonempty proper subset $Z \subset V$ such that $f(Z) \leq 0$ or

(b) a conclusion that $f(X) \geq 0$ for all $X \subseteq V$, i.e., $\mathbf{0} \in \mathrm{B}(f)$.

We denote the algorithm in Theorem 3 by Chubanov $(f)$.

Let us consider the complexity of $\operatorname{Chubanov}(f)$. Define an index $v$ by

$$
\begin{array}{r}
v=\quad \max \{\operatorname{vol}(W) \mid W: \text { a parallelopiped formed by } \\
\left.n-1 \text { vectors } w \in \mathrm{C}^{*}(\mathrm{~B}(f)) \text { and }\|w\| \leq 1\right\}, \quad \text { (13) }
\end{array}
$$

where $\operatorname{vol}(W)$ is the volume of $W$ in the Euclidean subspace $H_{0}=\left\{x \in \mathbb{R}^{V} \mid x(V)=0\right\}$ and $\|\cdot\|$ denotes the Euclidean norm. We can show that $\log \left(v^{-1}\right)=\mathrm{O}\left(n^{2} \log n M\right),{ }^{4}$ where $M=$ $\max \{|f(X)| \mid X \subseteq V\}$. Put $v^{*}=n^{2} \log n M$.

Due to Proposition 1, adapting Chubanov's theorem [4, Theorem 2.1] to our problem becomes as follows.

Theorem 4. Under Assumption (A $\left.\mathbf{A}^{*}\right)$ Algorithm Chubanov $(f)$ runs in $\mathrm{O}\left(\left(n^{5}+n^{3} T+n^{4} \mathbf{E O}\right) v^{*}\right)$ time, where $\mathbf{E O}$ denotes the time required for the function evaluation of $f$ and $T$ denotes the time required for computing a scalar $\gamma(x)$ such that $\gamma(x)^{2}\|x\|^{2} \in$ $\left[1, \frac{3}{2}\right]$, for extreme base $x \in \mathrm{B}(f)$.

\footnotetext{
${ }^{4}$ Replace the condition $\|w\| \leq 1$ in (13) by $\|w\|_{1} \equiv \sum_{v \in V}|w(v)| \leq 1$ to get a lower bound of $v$. Then use a fundamental fact [11, Theorem 3.2] on basic solutions of the associated LP. (A better bound $\mathrm{O}(n \log n M)$ is given by one of the anonymous reviewers with a proof, which improves the value of $v^{*}$ in Theorem 4.)
} 
It follows from Lemma 2 that $T=\mathrm{O}(\log n M)$.

Now consider the $\alpha$-enlargement $f^{(\alpha)}$ for $\alpha=\frac{1}{2}$ and perform Chubanov $\left(f^{(\alpha)}\right)$, or equivalently Chubanov $\left(2 \cdot f^{(\alpha)}\right)$ for integer-valued submodular function $2 \cdot f^{(\alpha)}$. Then it follows from Theorem 3 that we obtain

(a') a nonempty proper subset $Z \subset V$ such that $f(Z) \leq-1$ or

(b') a conclusion that $f(X) \geq 0$ for all $X \subseteq V$, i.e., $\mathbf{0} \in \mathrm{B}(f)$, where note that since $f$ is integer-valued, we have $f(Z) \leq-\frac{1}{2}$ if and only if $f(Z) \leq-1$ (and $f(X) \geq-\frac{1}{2}$ if and only if $f(X) \geq 0$ ).

This gives us a polynomial algorithm for testing membership $0 \in \mathrm{B}(f)$, which we denote by Membership $(f)$, and we obtain a set $X$ with $f(X)<0$ when $\mathbf{0} \notin \mathrm{B}(f)$.

\section{Submodular Function Minimization}

We have shown how the recent result of Chubanov [4] for linear programming yields a weakly polynomial algorithm Membership $(f)$ for testing membership $\mathbf{0} \in \mathrm{B}(f)$. However, this does not give any minimizer of $f$ in a direct way. Fujishige and Iwata [8] showed that by invoking Membership( $\cdot)$ as a subroutine $\mathrm{O}\left(n^{2}\right)$ times, we can find a minimizer of $f$.

In this section we show another way of finding a minimizer of $f$ by $\mathrm{O}\left(\log M_{*}\right)$ calls for the membership testing algorithm Membership( $\cdot)$, where $M_{*}$ is defined by (14).

First we make the following preprocessing.

- If $f(V)>0$, then put $f(V) \leftarrow 0$ for set $V$ alone. Then, function $f$ remains submodular and the set of minimizers (except for $V$ ) remains the same.

- If $f(V)<0$, then for all nonempty $X \subseteq V$ put $f(X) \leftarrow$ $f(X)-f(V)$. Then, function $f$ remains submodular and the set of minimizers (except for $\emptyset$ ) remains the same.

Put

$$
M_{*}=-\sum_{v \in V} \min \{0, f(V)-f(V \backslash\{v\})\} .
$$

Note that $0 \geq \min \{f(X) \mid X \subseteq V\} \geq-M_{*}$ due to Lemma 2. Now we employ Procedure Membership(·) as follows.

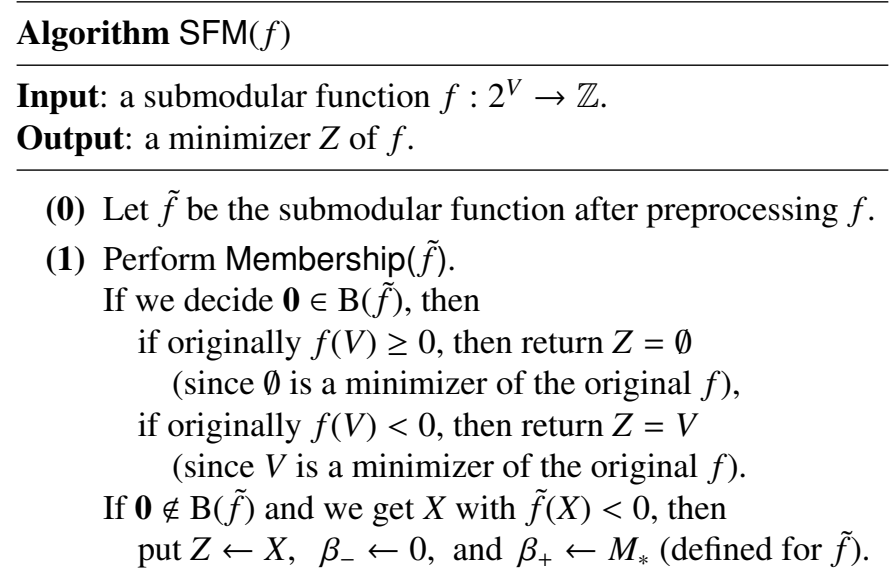

(2) While $\beta_{+}-\beta_{-} \geq 2$, do the following (i) and (ii):

(i) Put $\alpha \leftarrow\left\lceil\left(\beta_{-}+\beta_{+}\right) / 2\right\rceil$. (ii) Perform Membership $\left(\tilde{f}^{(\alpha)}\right)$.

If we decide $\mathbf{0} \in \mathrm{B}\left(\tilde{f}^{(\alpha)}\right)$, then put $\beta_{+} \leftarrow \alpha$. If $\mathbf{0} \notin \mathrm{B}\left(\tilde{f}^{(\alpha)}\right)$ and we get $X$ with $\tilde{f}^{(\alpha)}(X)<0$,

(3) Return $Z$

Theorem 5. Algorithm SFM $(f)$ returns a minimizer $Z$ of $f$ after repeating $\mathrm{Step}(2) \mathrm{O}\left(\log M_{*}\right)$ times.

Proof: The validity of Step (1) follows from the definition of preprocessing. While carrying out Step (2), we keep the property that $\mathbf{0} \in \mathrm{B}\left(\tilde{f}^{\left(\beta_{+}\right)}\right)$and $\mathbf{0} \notin \mathrm{B}\left(\tilde{f}^{\left(\beta_{-}\right)}\right)$. Hence, when we reach Step (3), we have $\tilde{f}^{\left(\beta_{-}\right)}(Z)<0 \leq \tilde{f}^{\left(\beta_{+}\right)}(X) \quad(\forall X \subseteq V)$ and $\beta_{+}-\beta_{-}=1$. This implies $-\beta_{+} \leq \tilde{f}(Z)<-\beta_{-}=-\beta_{+}+1$ and $-\beta_{+} \leq \tilde{f}(X)$ for all $X \subseteq V$. It follows that $Z$ attains the minimum of $\tilde{f}$ (and hence of $f$ ), which is equal to $-\beta_{+}$.

Combining Fujishige and Iwata's method [8] with SFM $(f)$, we can compute a minimizer of an integer-valued submodular function in $\mathrm{O}\left(\left(n^{5}+n^{3} T+n^{4} \mathbf{E} \mathbf{O}\right) v^{*} \min \left\{n^{2}, \log M_{*}\right\}\right)$ time.

\section{Concluding Remarks}

It should be noted that when we perform Membership $(f)$ and decide $\mathbf{0} \in \mathrm{B}(f)$, we do not have a certificate of $\mathbf{0} \in \mathrm{B}(f)$ by an expression of $\mathbf{0}$ as a convex combination of (extreme) bases in $\mathrm{B}(f)$. Because of this it seems that applying Chubanov's algorithm does not directly give us a dual certificate for a minimizer of $f$.

\section{References}

[1] R. E. Bixby, W. H. Cunningham and D. M. Topkis: Partial order of a polymatroid extreme point. Mathematics of Operations Research 10 (1985) 367-378.

[2] S. Chubanov: A strongly polynomial algorithm for linear systems having a binary solution. Mathematical Programming, Ser. A 134 (2012) 533570.

[3] S. Chubanov: A polynomial projection algorithm for linear feasibility problems. Mathematical Programming, Ser. A 153 (2015) 687-713.

[4] S. Chubanov: A polynomial algorithm for linear feasibility problems given by separation oracles. Optimization Online, March 2017.

[5] D. Dadush, L. A. Végh, and G. Zambelli: Rescaling algorithms for linear programming Part I: Conic feasibility. arXiv:1611.06427v1 [math.OC] 19 Nov 2016.

[6] D. Dadush, L. A. Végh, and G. Zambelli: Geometric rescaling algorithms for submodular function minimization. Proceedings of the 29th SODA (2018) 832-848.

[7] S. Fujishige: Submodular Functions and Optimization Second Edition (Elsevier, 2005).

[8] S. Fujishige and S. Iwata: A descent method for submodular function minimization. Mathematical Programming, Ser. A 92 (2002), 387-390.

[9] S. Iwata, L. Fleischer, and S. Fujishige. A combinatorial strongly polynomial algorithm for minimizing submodular functions. Journal of the ACM 48 (2001) 761-777.

[10] Y. T. Lee, A. Sidford, and S. C.-W. Wong: A faster cutting plane method and its implications for combinatorial convex optimization. Proceedings of 56th Annual Symposium on Foundations of Computer Science FOCS2015 (2015) 1049-1065.

[11] A. Schrijver: Theory of Linear and Integer Programming (Wiley, 1986).

[12] A. Schrijver: A combinatorial algorithm minimizing submodular functions in strongly polynomial time. Journal of Combinatorial Theory, Ser. B 80 (2000) 346-355. 